\title{
Public Large Screen enabled Content Collection and Connection
}

\author{
Kosuke Numa ${ }^{1}$, Hironori Tomobe ${ }^{1}$, Tatsuo Sugimoto ${ }^{1}$, Masako Miyata $^{2}$, \\ Kiyoko Toriumi ${ }^{1}$, Jun Abe ${ }^{1}$, and Koichi Hori ${ }^{1}$ \\ 1 The University of Tokyo, 4-6-1 Komaba, Meguro, Tokyo, Japan, \\ 2 Sapporo Otani University, 1-1 Kita 16, Higashi 9, Higashi, Sapporo, Japan \\ numa@ai.rcast.u-tokyo.ac.jp
}

\begin{abstract}
In this paper, we propose a framework for content collection and connection enabled by public large screens and mobile phones. Making people express their stories will encourage their active attitudes toward information management. With the proposed framework, we aim to overcome difficulties on managing flooding information. We applied the framework on two practice oriented systems and held workshops using them.
\end{abstract}

\section{Introduction}

Nowadays, we people are living in rapidly increasing quantity of information and feeling difficulties in managing them. A new way is required to manage this flooding information in the coming intelligent society.

We consider that one good way to manage information is to publish new information. To publish their own thoughts, people need to collect related information, think over them, create new relations among them, and add their own opinions. A publishing process requires whole activities around information management. Blogging is one example of such practices. It changed people's attitudes toward information on the Web. In this research, we aim to design new relationship between people and information in the real world. To manage everyday information, people need to express their stories and exchange them. Moreover, a way to connect stories, not just to collect stories, is required since people's stories are not separately concluded.

In this paper, we propose a framework for content collection and connection enabled by large screens and mobile phones. We develop two types of practice oriented systems and organize workshops using them. This research does not provide thorough solution to whole design of social interaction with information in the real world; we show a pilot design of relationship between contents and people. In the next section, we describe the background of this research and our framework. Based on the framework, we show two practices in Sections 3 and 4. In Section 5 we provide discussions and we conclude this paper in Section 6 . 


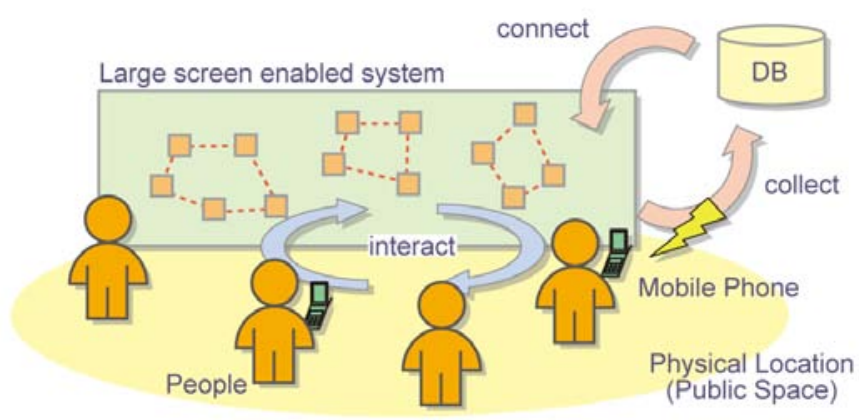

Fig. 1. Proposed framework for content collection and connection

\section{Large Screen-enabled User-contributed Systems}

Storytelling or narrative approaches are getting widely accepted in several fields such as psychology, folklore, education, and therapeutics. It is said that people articulate and interpret their temporal experience by telling stories[7,8]. Storytelling helps people to understand and manage their experiences. First, we aim to make ordinary people tell their stories.

We consider that stories exist not separately but within relations among each other. In hypertext systems like WWW, contents are connected to others. Connected stories are weaved up to larger stories. Second, we aim to connect collected stories.

We employ mobile phones as expressing tools. Recently, mobile phones are usually equipped with cameras. People can take photos and videos and can send them to their friends with their mobile phones. Moreover, some people upload their photos to their blogs or photo sharing sites from their phones. Now, with our mobile phones, we can exchange our experience everywhere in real time ideally. But most of mobile contents are separately concluded.

We propose a framework to connect and collect people's stories. Figure 1 illustrates outline of the framework. A large screen enabled user contributed system is installed in a public space where people can freely access. People publish their stories using mobile phones. The system stores collected contents and connects them. Connected stories are projected on the screen and people there can interact with them. People's stories form larger stories and return to people. People can change presented connections by adding new contents. In the system, people's stories are iteratively weaved up.

We will find digital signages everywhere in near future[3,2]. They can be shared places for content creation and connection. Peltonen and his group developed an interactive collaboration system using a multi-touch large screen and mobile phones[6]. Their research is technically similar to this research, but we aim at content connections rather than direct interactions among participants. 


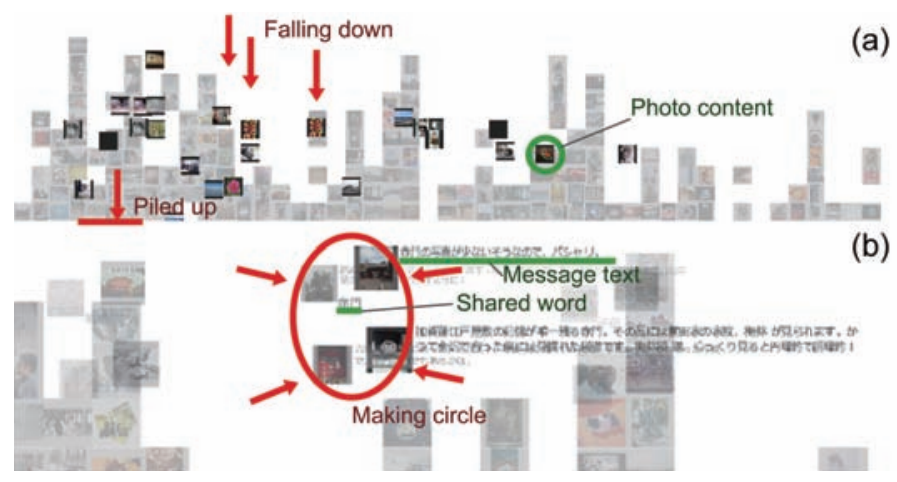

(a)

Fig. 2. Screenshots of the shower-style view

In this paper, we describe our two workshops which were designed based on this framework. A term workshop here we mean a specially managed place for practice where people gather and act collaboratively. We regard a workshop itself as a creativity support system[4]. Our final purpose is to redesign relation between people and contents in everyday lives - not in closed spaces like workshops. But the field practices will provide clues to this purpose.

The first practice is the collective photo collage workshop[5]. We collected photo-attached mobile messages and projected them on a screen in a public space. Contents are automatically connected based on their text. The second practice is the mobile video workshop[1]. We collected and presented mobile videos which record interviews connected by question and answer game. In this practice, contents themselves have connections to other contents. We describe the first workshop in Section 3 and the second in Section 4 respectively.

Our purposes in this research are to draw people's stories and to connect them. It is fundamentally important for people to know that their own stories affect whole stories - to know that one is a part of the world. Our practice is designed to simplify and to emphasize this point.

\section{Collective Photo Collage Workshop}

The first workshop is a pilot practice for collecting and connecting mobile pictures. We developed a system which stores photo-attached messages and automatically connects them. In this section, we introduce this practice briefly.

The workshop was held at a new hall in the University of Tokyo in Japan, which was built on March, 2008. In the workshop, we collected photo-attached e-mails from people around/related to the hall. Collected contents are connected based on shared words included in messages. Messages are morphologically analyzed and shared words are automatically extracted. The system stores contents (photos and text messages) and these data (extracted words) to a database. 


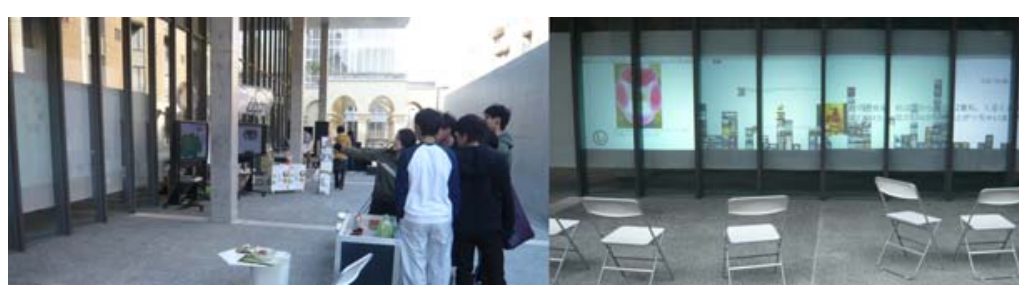

Fig. 3. Scenes from the collective photo collage workshop

Collected contents were shown on two views on site (the slide show view and the shower-style view) and on the Website. The shower-style view connects messages based on shared words. Figure 2 shows screenshots of the shower-style view. Collected contents fall down from the upper edge of the screen and are piled up on the bottom edge (Figure 2(a)). After all entries are piled up, several entries form a circle based on a randomly selected shared word (Figure 2(b)). Minimum number of entries for each circle is four.

As shown in Figure 3, the slide show view and the shower view are projected onto the screen. Passengers can see entries on the screen, and can post their own entries on site with their mobile phone. The posted entries are soon displayed on the slide show, and will be used for word-based circles.

We began to collect contents online from 22nd February 2008. The workshop was held 21st through 28th March. Through the event, 351 entries are posted by 184 users. Since the detailed analysis is described in [5], here we introduce just several findings. There were roughly two types of participants: participants who posted multiple contents online, and participants who posted single contents for each from the venue. While $88.6 \%$ of contents were connected to other contents, participants on site tended to communicate with people/things there rather than to interact with contents on the system. The aim that connected stories form large stories was not fully achieved. A participant also claimed this point - "I want to put my photo directly into a circle."

\section{Mobile Video Workshop}

Contents were not directly connected in the first workshop; connections were generated automatically. We devised the system to show direct connections.

\subsection{Outline}

We designed a workshop program called "Keitai Trail?" and practiced it in conjunction with Ars Electronica festival 2008. Ars Electronica festival is one of the most popular media art festival held annually in Linz, Austria. A word Keitai is a mobile phone in Japanese.

In this workshop, we record people's stories on mobile videos and connected them directly based on a rule. Figure 4 illustrates the workshop scenario (and the 


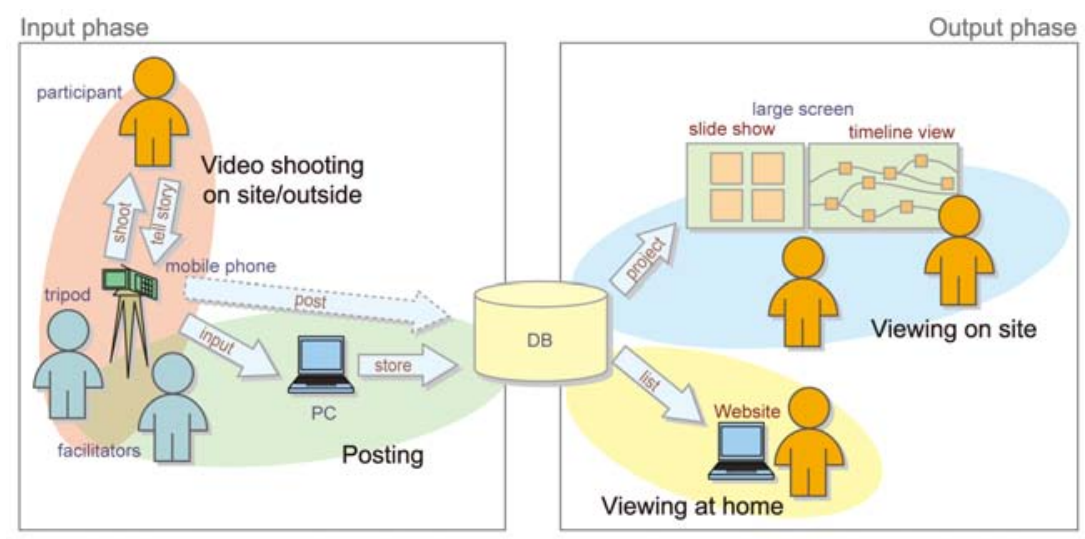

Fig. 4. Workshop scenario and system architecture

system architecture explained in the next subsection). This workshop is designed to be held not only at a single place but also outside space around a main venue.

The main venue is a kind of base where facilitators present a progress and participants' expressions of the workshop. Facilitators go outside and ask people there to join the workshop. If one accepts, facilitators shoot a video of her talk with a mobile phone. In the main venue, connected videos are shown on large screens by an installed support system which we describe in the following section.

Participants' talks are requested to follow the "talking format" shown in Figure 5. The format connects talks in a simple rule. The format consists of four parts: (1) an answer to a question from a former participant, (2) a short free talk, (3) a connecting phrase to the next part, and (4) a question to a next participant. The question in the fourth part will be answered in a next participant's first part. This is like a question and answer game. A question from a former participant is a cue to a free talk; a talk in the second part is a core of her story. Participants can choose a question to answer, i.e., her former participant. With this rule, a story is connected to other stories. Connected stories make large stories. This format derives people's stories and connects them. Figure 6 shows example stories and their connections.

\subsection{System}

Figure 4 illustrates a usage scenario of the installed system which consists of two phases.

In input phase, facilitators shoot a video of a participant telling story with a tripod equipped mobile phone. Facilitators store video files and their connections to a database. It is theoretically possible to post videos directly from mobile phones. But in this case, we needed to develop an input interface for PCs due to temporal technological limitations such as maximum size of uploading files and covered service in roaming area of mobile phones we used. 


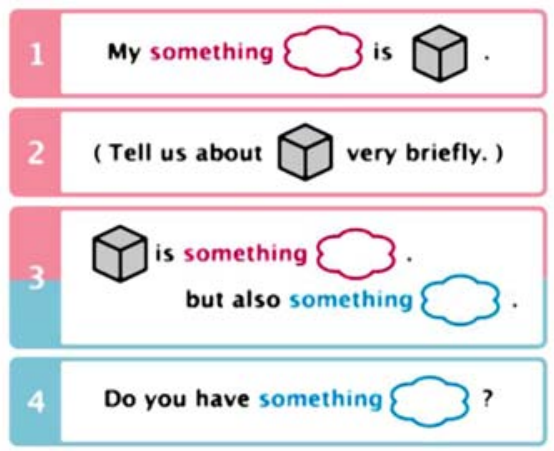

Fig. 5. Talking format we used in our practice

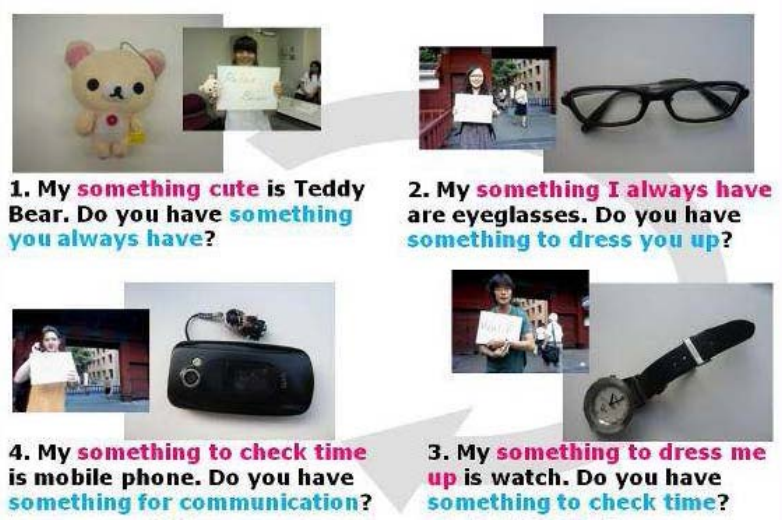

Fig. 6. Example stories and their connections

In the output phase, we prepared two ways of viewing. At the main venue, two types of interfaces are projected on large screens. Slide show view plays recently posted two and randomly selected two videos at a same time. Timeline view is designed to show whole connections of videos (Figure 7). In the view, nodes represent videos and arcs represent connections. The x-axis direction stands for time and the view can be scrolled in this direction. A participant can trace whole stories and can add her story. Participants who joined outside the venue have two options to see their own videos. They can visit the venue of course. In addition, we provide a Website which lists collected videos. They can browse stories at home.

\subsection{Result}

We held the workshop during 4th to 9th September 2008 in Linz, Austria. Finally, we collected 258 stories (videos): 218 in this workshop and 40 in the preliminary workshop held in Japan. Scenes from the workshop are shown in Figure 8 


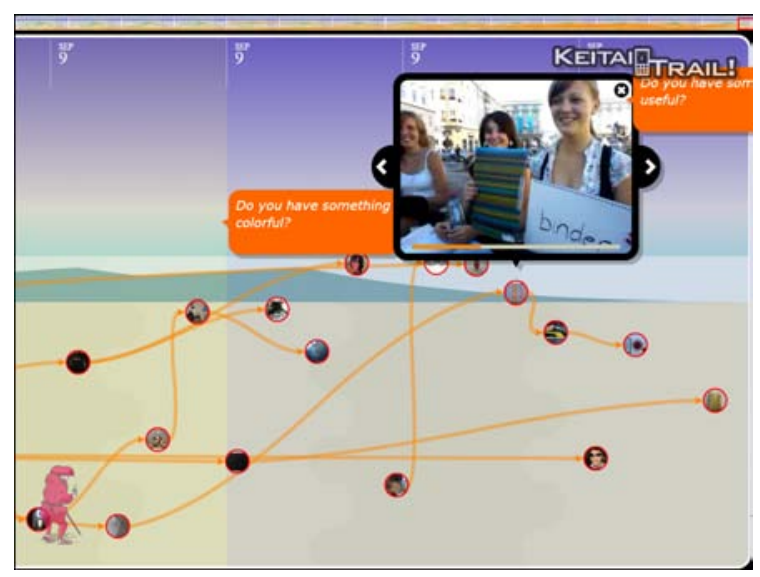

Fig. 7. Screenshot of timeline view

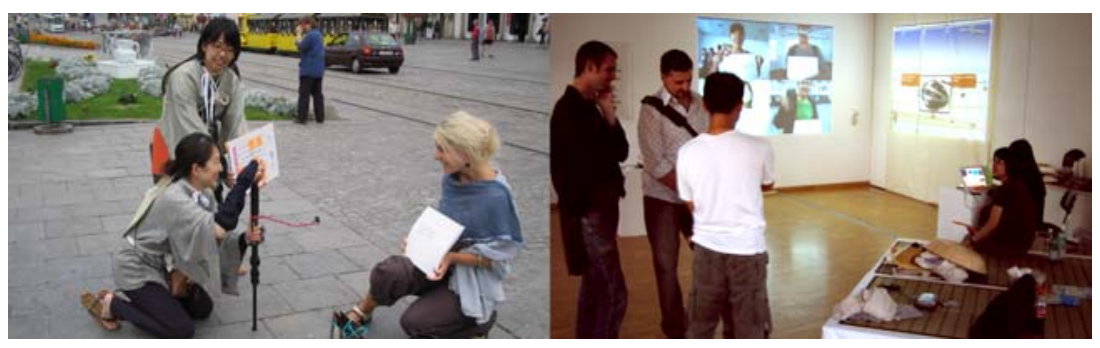

Fig. 8. Scenes from the mobile video workshop (the venue and outside)

These videos are divided into five clusters. The largest cluster includes 174 videos, and has 28 branches. The longest path in the cluster is 38 connections (39 videos) in length. Most of stories are connected to this cluster or the second largest cluster including 77 videos. The other three clusters were cut off from the larger clusters by a few participants who ignored former contents. Most of stories occupy parts of large stories.

\section{Discussion}

Through two workshops, we connected contents. In the first workshop, connections were generated automatically. In the second workshop, we designed the system to connect contents directly and manually. As a result, we obtained more active commitment from participants in the latter workshop.

These two workshops do not differ so much technically. Contents are stored to databases, the contents and their connections are output in XML data. Interfaces read the data and present them in designed views. Interactions in the workshops, however, were different. While a content and its relation could not be seen just 
after it was posted in the former workshop, we showed direct connections of contents in the latter workshop. One of the reasons for the difference of the results was this difference of interaction design.

\section{Conclusion}

In this paper, we proposed a framework for content collection and connection enabled by public large screens and mobile phones. We applied the framework on two practice oriented systems and held workshops using them. Through this paper, we wanted to claim that expressing a single story is a small activity, but it has a big meaning socially.

\section{Acknowledgements}

This work has been supported by a grant from the Japan Science \& Technology Agency under CREST Project.

\section{References}

1. Jun Abe, Kiyoko Toriumi. Collaborative Narratives in the Digital Age: An Analysis of "Keitai Trail! - Mobile Video Workshop -". In Proceedings of Annual Workshop on Digital Communication, 2009.

2. Dagmar Kern, Michael Harding, Oliver Storz, Nigel Davis, and Albrecht Schmidt - Shaping How Advertisers See Me: User Views on Implicit and Explicit Profile Capture. In CHI'08 Extended Abstracts, 2008.

3. Jörg Müller, Alex Schlottmann, and Antonio Krüger. Self-optimizing Digital Signage Advertising. In Adjunct Proceedings of Ubicomp2007, 2007.

4. Kosuke Numa, Kiyoko Toriumi, Katsuaki Tanaka, Mina Akaishi, and Koichi Hori. Participatory Workshop as a Creativity Support System. In 12th International Conference on Knowledge-Based and Intelligent Information $\mathcal{E}$ Engineering Systems (KES2008), 2008.

5. Kosuke Numa, Hironori Tomobe, Katsuaki Tanaka, Takuichi Nishimura, Koichi Hori, and Takeshi Sunaga. A Case Study on Interactions with User Contributed Website in Public Space. In The 7th International Workshop on Social Intelligence Design (SID2008), 2008.

6. Peter Peltonen, Antti Salovaara, Giulio Jacucci, Tommi Ilmonen, Carmelo Ardito, Petri Saarikko, and Vikram Batra. Extending Large-scale Event Participation with Usercreated Mobile Media on a Public Display. In Proceedings of the 6th International Conference on Mobile and Ubiquitous Multimedia, 2007.

7. Paul Ricœur. Temps et Récit. Seuil, 1983. (Time and Narrative. trans. Kathleen McLaughlin and David Pellauer. University of Chicago Press, 1984.)

8. Roger C. Schank. Tell Me a Story: Narrative and Intelligence. Northwestern University Press, 1990. 